\title{
变截面纳米线声子输运蒙特卡洛模拟
}

\author{
王赞 ${ }^{1}$, 赵睿杰 ${ }^{(1)}$, 陈云飞 ${ }^{(1) *}$ \\ (1) 东南大学机械工程学院机械设计系, 南京 211189 ; \\ (2) 东南大学教育部 MEMS 重点实验室, 南京 210096 \\ *E-mail: yunfeichen@seu.edu.cn
}

收稿日期: 2009-03-09; 接受日期: 2009-07-19

国家教育部博士点基金(批准号: 20050286019)和国家自然科学基金(批准号: 50676019)资助项目

\begin{abstract}
摘要采用蒙特卡洛(MC)方法建立了声子在变截面硅纳米线内的输运模型, 以渐扩几何体与 渐缩几何体为物理模型, 分别研究了在等热量与非等热量输入条件下 2 种模型的热整流效应和声 子的输运特性. 模拟结果表明在 2 种条件下, 渐缩模型的热传导性能均高于渐扩模型. 在非等热 量输入的条件下, 渐缩模型的热整流效应要大于等热量输入条件下的热整流效应. 2 种物理模型 的主要区别在于体积的分布不同, 虽然在纳米尺度下边界散射作用明显, 但模拟结果表明边界散 射对热传导的影响小于质量分布不同对声子输运的影响.
\end{abstract}

关键词

蒙特卡洛

纳米线

变截面

热整流
当传热器件的几何特征尺寸减小至纳米尺度, 由于量子效应、边界效应的作用使其具有与宏观结 构相差较大的物理特性. $\mathrm{Li}$ 等人 ${ }^{[1]}$ 采用汽液固相 (vapour-liquid-solid, VLS)方法制备了硅纳米线, 测得热 导率与体态硅相比降低了至少 2 个数量级. Hochbaum 等人 ${ }^{[2]}$ 通过水化学刻蚀(electroless etching, EE)方法获得 硅纳米线, 结果显示热导率为VLS 方法的 1/5 1/8. 近 10 年来, 大多数研究侧重于等截面纳米结构的热传 导, 关于变截面纳米结构热传导的研究报道并不多. 与等截面结构相比, 变截面结构沿某一个方向的边 界条件和质量分布是变化的, 因而对声子输运的影 响有所不同. Chang 等人 ${ }^{[3]}$ 将纳米碳管一端沉积 $\mathrm{C}_{9} \mathrm{H}_{16} \mathrm{P}_{\mathrm{t}}$ 材料形成非对称的几何外形, 实验数据表明 改变热流方向, 热导相差 $7 \%$. Wu 等人 ${ }^{[4]}$ 采用分子动 力学(molecular dynamics, MD)方法研究了 $(n, 0) /(2 n, 0)$ 碳纳米管结, 热整流效率能够达到 $20 \%$. Yang 等人 ${ }^{[5]}$ 采用 MD 方法研究了 200 400 K温度范围内碳纳米雉
的热整流效应, 结果表明碳纳米雉的非对称几何结 构产生的热整流效应非常明显. 变截面纳米结构的 热整流效应说明在纳米尺度下热传导性能不仅和结 构尺寸有关而且与质量分布有关. 在过去的几十年 里, 电整流、二极管和晶体管的发明推动了电子器件 的研究和应用, 然而至今还未出现有实际应用价值 的热整流、热二极管和热晶体管. 如果能对热流进行 有效管理, 声子将像电子和光子一样成为传递信息 的载体, 那么声子器件将具有非常广阔的应用前景.

研究纳米尺度下声子输运的方法可分为解析法 与数值法. 玻尔兹曼输运方程 (Boltzman transport equation, BTE)通常作为理论分析声子输运的起点. 解析法中, 一种途径是将边界散射作为散射项引入 BTE方程, 另一种途径是改变声子色散曲线 ${ }^{[6]}$. 2 种 途径的共同点在于改变了声子在纳米结构中的自由 行程. 数值方法包括分子动力学方法与 $\mathrm{MC}$ 方法等. MD方法 ${ }^{[7]}$ 通过求解各粒子在相互作用下的运动方程,

引用格式: Wang Z, Zhao R J, Chen Y F. Monte Carlo simulation of phonon transport in variable cross-section nanowires. Sci China Tech Sci, 2010, 53: 429-434, doi: 10.1007/s11431-009-0338-3 
统计每个粒子的空间位置、运动状态, 得到材料宏观 行为特征. 然而当模型包含大规模粒子时, MD方法 计算效率低小, 限制了其应用范围. MC方法的原理 是将原子热振动量子化为声子, 原子间热振动耦合 转化为声子间散射, 从而无需计算原子受力与运动 方程, 在很大程度上降低了计算量. 在模拟中, 经过 足够长的时间演进, 系统达到平衡态后, 从中抽取一 定数量的样本可以获得系统状态参数与声子分布. 近几年来研究者 ${ }^{[8 \sim 11]}$ 将 $\mathrm{MC}$ 方法应用于研究纳米薄 膜、纳米线中的声子输运, 获得了比较理想的结果, 并在模拟数据与实验数据对比的基础上, 分析了等 截面纳米结构中影响热传导的关键因素. 然而, 目前 国内外很少有文献介绍变截面纳米线的热传导特性 与热整流效应, 本文旨在通过蒙特卡罗算法(Monte Carlo, MC)研究变截面条件下声子的传输过程, 分析 热整流效应的发生机理与边界效应对变截面模型的 影响, 为进一步提高纳米结构材料的热电品质系数 探索新方法.

\section{1 声子在半导体中的输运过程}

声子在半导体中的输运过程经历漂移与散射 2 个阶段: 在漂移阶段声子沿直线运动, 极性、频率与 速度均不发生改变; 当发生边界散射、杂质散射或声 子间散射时, 声子终止漂移阶段进入散射阶段. 边界 散射与杂质散射均为弹性散射, 声子极性与频率保 持不变. 速度方向将发生改变, 从而引起声子沿热流 方向上的分速度重新分配, 对热流产生影响. 声子间 散射主要研究三声子散射即 Normal (N) 散射与 Umklapp (U)散射 ${ }^{[12]}$. 对于 $\mathrm{N}$ 散射, 当 2 个声子合成 1 个声子或 1 个声子分解成 2 个声子时, 散射前后保持 能量守恒与动量守恒. 从系统整体角度考虑, $\mathrm{N}$ 散射不 会改变声子系统的总能量与总动量, 在声子均匀分 布的情况下不会对热流产生阻碍, 因而在模拟程序 中不考虑 $\mathrm{N}$ 散射对热导的影响. $\mathrm{U}$ 散射过程中, 当两 声子发生合成散射时, 若两者波矢的矢量和超越布 里渊区的正边界, 则新声子的波矢为两者矢量和减 去一个倒格矢, 使其落入布里渊区的负区间, 从而新 声子的速度方向从正向改变为负向, 反之亦然. 在温 度梯度作用下, 沿热流方向运动的声子要多于反方 向运动的声子, 因此 $\mathrm{U}$ 散射对热流将产生直接的阻 碍作用.

\section{MC模型}

声子在体态导热体内输运, 由于几何尺寸远远 大于声子平均自由行程, 所以主要的散射模式是声 子间散射与杂质散射. 并且, 由于体态导热体在三维 方向具有较大的尺寸使得声子波矢在第一布里渊区 内近似连续分布. 相比之下, 由于纳米结构导热体在 至少一个方向上达到纳米尺度, 使声子与导热体表 面发生边界散射的几率增大, 引起热导率大幅度降 低. 图 1 所示为声子在变截面纳米线中输运的示意图, 假设模型的所有表面均为绝热面, 模型等分为 7 个单 元, 每个单元的长度为 $50 \mathrm{~nm}$, 厚度为 $50 \mathrm{~nm}$, 宽度随 热流方向线性变化. 纳米线两端分别为热浴与冷浴, 声子由热浴进入, 从冷浴流出. 在一个循环周期内, $\mathrm{MC}$ 模型经过初始化、声子漂移、声子散射、统计单 元声子状态 4 个步骤后开始新的循环. 一个循环周期 消耗的时间用一个步长时间表示, 步长时间的设置 应该足够小以免错过任何一种散射事件. 若步长时 间过大，不仅增大误差而且在边界面交汇处易形成 死区即声子在此区域内与多个表面发生边界散射无 法逃出, 导致程序进入死循环. 经过多次调试, 发现 当最高速的声子在一个步长时间内漂移的距离为 $1 / 5$ 单元长度时, 程序计算效率与模拟结果较为理想.

在模拟开始时, 需要初始化模型中各单元声子 数, 速度和位置. 声子服从玻色-爱因斯坦分布, 平衡 态条件下当温度已知时, 单位体积内频率为 $\omega$ 的声 子数目可由下式得到

$$
n_{\omega}=1 /\left(e^{\hbar \omega / k T}-1\right),
$$

式中, $n_{\omega}$ 为声子数目, $=h / 2 \pi, h$ 为普朗克常量, $k$ 为

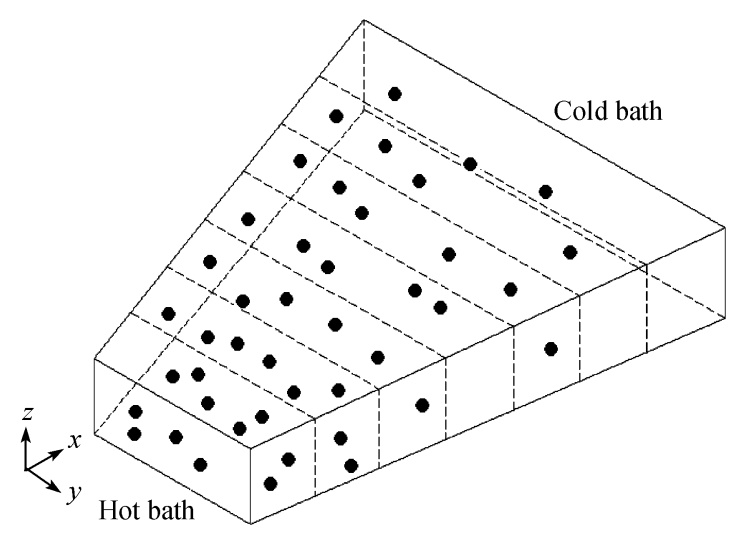

图 1 变截面纳米线热传导物理模型 
玻尔兹曼常量, $T$ 为温度. 根据体态硅 $\langle 001\rangle$ 方向的色 散关系 ${ }^{[11]}$, 声子具有光学与声学 2 个分支. 由于光学 分支声子群速度较小, 对热传导影响几乎可以忽略, 计算中仅考虑声学分支. 首先将色散曲线的截止频 率均分为 1000 个频段, 通过下式计算出传热单元的 纵波声子与横波声子的数量、速度及位置分布.

$$
D(\omega)=\frac{K^{2}}{2 \pi^{2}} \frac{\mathrm{d} K}{\mathrm{~d} \omega},
$$

$D(\omega)$ 表示单位频率间隔内包含的模式数目.

$$
\begin{aligned}
N= & \sum_{i=1}^{1000} n\left(\omega_{i}, \mathrm{LA}\right) D\left(\omega_{i}, \mathrm{LA}\right) \Delta \omega_{i} V \\
& +2 \sum_{i=1}^{1000} n\left(\omega_{i}, \mathrm{TA}\right) D\left(\omega_{i}, \mathrm{TA}\right) \Delta \omega_{i} V,
\end{aligned}
$$

$N$ 表示总声子个数, 等式右边第 1 项与第 2 项分别表 示第 $i$ 个频段所包含的纵波声子与横波声子数目. $D\left(\omega_{i}\right.$, LA)表示第 $i$ 个频段, 纵波声子的态密度. $D\left(\omega_{i}, \mathrm{TA}\right)$ 表示第 $i$ 个频段, 横波声子的态密度. $V$ 表示传热单元 体积, $\Delta \omega_{i}$ 表示频段间隔. 获得传热单元声子的数量 后，通过下式对声子速度与位置进行赋值

$$
V_{i}=\nabla_{k_{i}} \omega_{i},
$$

式中, $V_{i}$ 为第 $i$ 个频段声子群速度.

$$
\begin{aligned}
{\left[V_{X}, V_{Y}, V_{Z}\right]=} & V_{i}\left[\cos \left(2 \pi R_{1}\right), \sin \left(2 \pi R_{1}\right) \sin \left(2 \pi R_{2}\right),\right. \\
& \left.\sin \left(2 \pi R_{1}\right) \cos \left(2 \pi R_{2}\right)\right],
\end{aligned}
$$

式中, $V_{X}$ 表示声子 $X$ 方向分速度, $V_{Y}$ 表示 $Y$ 方向分速 度, $V_{Z}$ 表示 $Z$ 方向分速度, $R_{1}$ 与 $R_{2}$ 均为独立的随机数.

$$
\left[P_{X}, P_{Y}, P_{Z}\right]=\left[L_{X} r_{1}, L_{Y} r_{2}, L_{Z} r_{3}\right],
$$

$P_{X}, P_{Y}, P_{Z}$ 分别为声子在 $X, Y, Z$ 坐标分量, $L_{X}, L_{Y}, L_{Z}$ 为 单元在 3 个方向上的几何长度. 模型中 $L_{X}$ 与 $L_{Z}$ 为常 量, $L_{Y}$ 为变量. $r_{1}, r_{2}, r_{3}$ 均为独立随机数. 声子经过一 个步长时间到达新的位置, 首先需要判断是否超越 边界, 再判断是否发生杂质散射或声子间散射. 边界 散射存在镜面反射与漫反射 2 种情况, 当发生镜面反 射时不会改变声子沿热流方向的速率, 漫反射时将 发生改变. 图 2 显示了声子在变截面模型 $X-Y$ 方向发 生边界散射的二维示意图, 粗实线 $C D$ 为边界面, $P O$ 垂直于热流方向. $P O^{\prime} \perp C D, \alpha$ 为边界面倾角, $\beta$ 为 入射角. $A P$ 为声子在 $X-Y$ 平面的入射速度, $P B$ 为反射 速度. 以 $P$ 为圆心以 $A P$ 为半径做半圆交边界面与 $C$, $D$ 两点, $B$ 点落在 $C D$ 之间半圆上任意一点的概率相 等. 假设 $\angle A P O=\angle B P O=\beta$, 则声子沿热流方向的速

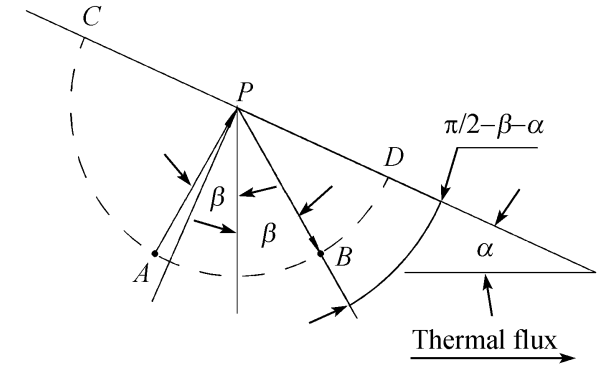

(a)

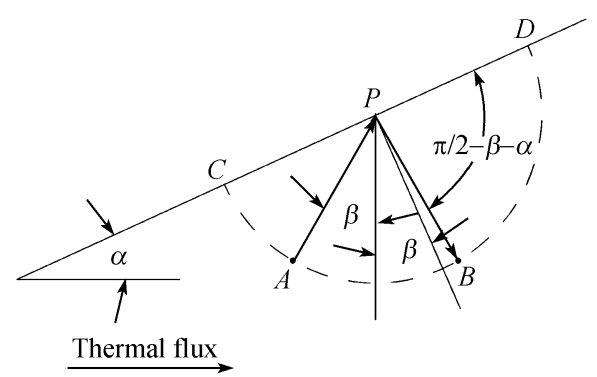

(b)

图 2 边界散射示意图

(a) 渐缩模型; (b) 渐扩模型

度分量不发生改变. 若 $B$ 点落在弧 $\overparen{D B}$, 热流方向上 的分速度将增加. 若 $B$ 点落在弧 $\overparen{B A C}$ 上则热流方向 上的分速度将减小. 图 2(a)中声子发生边界散射后获 得减速的概率为: $P_{1}=(\pi / 2-\beta-\alpha) / 2 / \pi$. 图 2(b)中声子获 得加速概率为: $P_{2}=(\pi / 2-\beta+\alpha) / 2 / \pi$. 由于 $P_{1}>P_{2}$, 故边 界对渐扩模型的声子起加速作用, 对渐缩模型起减 速作用. 边界散射的处理可以先按照等截面模型边 界处理方法 ${ }^{[11]}$ 计算出反射后的位置坐标与速度分量, 然后乘以旋转矩阵获得变截面条件下的位置与速度. 对于杂质散射和 $\mathrm{U}$ 散射, 假设声子的频率与极性不 变, 仅改变速度方向, 从而可以保证系统能量守恒避 免调温过程. 当完成一个步长时间后, 需要统计各单 元声子的极性、速度、频率和位置, 然后对其重新标 号进入下一个循环. 选取 2 个单元截面, 完成足够多 的循环后, 通过两截面的热流量之差小于 $10^{-20} \mathrm{~J}$ 即频 率最高声子的能量时, 认为系统达到平衡. 按照下式 统计出各单元的能量

$$
E=V \sum_{p} \sum_{i}^{1000}\left(n\left(\omega_{i}, p\right)+\frac{1}{2}\right) \hbar \omega_{i} D\left(\omega_{i}\right),
$$

式中, $E$ 为单元能量, $p$ 表示声子极性, 单元温度可通 过二分法获得. 


\section{3 模拟结果与讨论}

为了验证模型的可靠性, 先对等截面纳米线进 行模拟. 散射类型包括边界散射、杂质散射与 $U$ 散射, 相关参数与 Chen 等人 ${ }^{[7]}$ 选取一致. 为了获得较好的 统计结果, 各单元模拟声子个数不小于 5000 个, 统 计步数为 100 万步. 如图 3 所示, 纵坐标为热导率, 横 坐标表示温度即: $T=\left(T_{\mathrm{h}}+T_{\mathrm{c}}\right) / 2, T_{\mathrm{h}}$ 为热浴温度, $T_{\mathrm{c}}$ 为冷 浴温度. 中间单元的初始温度设置为 $T_{\mathrm{c}}$. 随着温度的 升高, 由于高频声子在声子总数中所占的比例逐渐 增加, 导致热浴与冷浴间声子数量差逐渐减小. 因而 需要随着模拟温度的升高, 加大热浴与冷浴之间温 差降低统计误差. 模拟中, 当 $T \leqslant 100 \mathrm{~K}$ 时, 温差设置 为 $10 \mathrm{~K}$. 当 $T>100$ 时, 温差为 $20 \sim 40 \mathrm{~K}$. 圆形标记为实 验数据, 方形标记为模拟数据. 模拟数据表明, 本文建 立的 MC 模型对边界散射、杂质散射、 $\mathrm{U}$ 散射的处理能 够较好地用于模拟声子在纳米线中的散射过程.

图 4 所示为变截面纳米线几何模型的俯视图, $L_{\mathrm{b}}$ 表 示模型大端宽度, $L_{\mathrm{s}}$ 表示小端宽度, $\mathrm{Z}$ 轴方向为 $50 \mathrm{~nm}$ 等 厚度. 在图 4(a)中, 热浴设置在模型大端, 冷浴设置在 模型小端(记为模型 A), 图 4(b) 与之相反(记为模型 B).

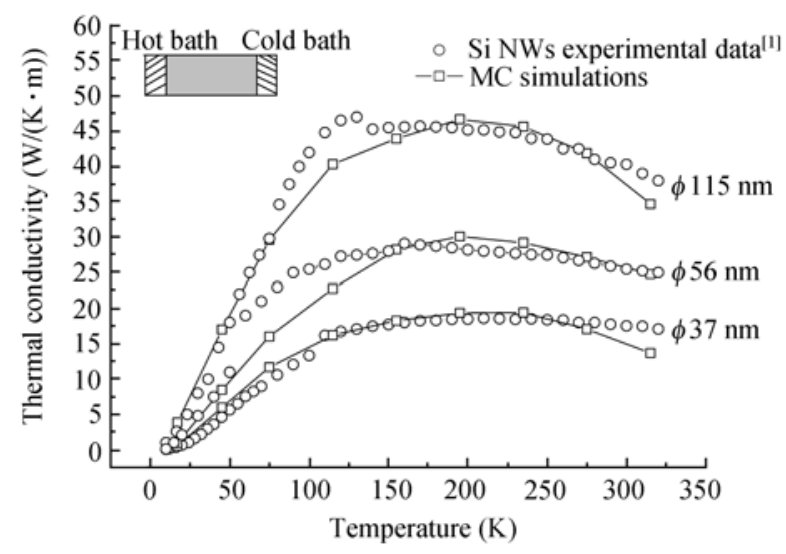

图 3 等截面硅纳米线热导率

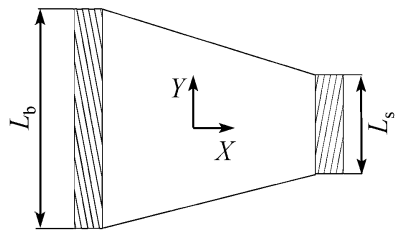

(a)

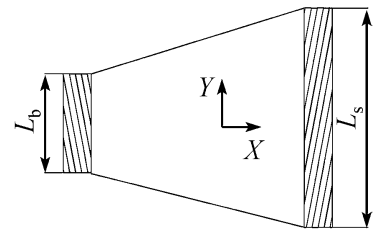

(b)
图 4 变截面纳米线示意图(非等能量输入)

(a) 热浴设置在大端，冷浴设置在小端; (b) 热浴设置在小端, 冷浴设置在大端
为了研究在相同温度梯度下，以上 2 种模型温度 分布差异, 将热浴温度设置为 $50 \mathrm{~K}$, 冷浴温度设置为 $40 \mathrm{~K}$, 边界散射设置为全部漫反射. 经过 100 万个步 长时间的模拟, 获得如图 5 所示沿 $X$ 轴方向的温度分 布, 与等截面纳米线相比, 温度曲线呈现出非线性变 化. 图 5 中共有 7 组数据, 前 3 组由模型 $\mathrm{A}$ 获得, 温 度分布呈非线性分布，并且非线性随热端与冷端的宽 度差的增加而增强, 插值曲线表现为下凸; 第 4 组数 据由矩形等截面模型获得, 温度分布呈线性分布; 后 3 组由模型 $\mathrm{B}$ 获得, 与前 3 组数据类似, 随着热端与冷 端宽度差的增加非线性增强，插值曲线表现为下凹. $\mathrm{CaO}$ 等人 ${ }^{[13]}$ 采用 $\mathrm{MD}$ 方法获得了类似的温度分布特性.

由图 5 可知, 交换热浴和冷浴的位置能够引起传 热性能上的改变. 为了研究 2 种模型热传导差异, 将 冷浴温度控制在 $100 \mathrm{~K}$, 热浴温度从 $110 \mathrm{~K}$ 逐渐升高 至 $310 \mathrm{~K} . \Delta T_{\mathrm{H}}$ 表示热浴的温度升高量, $\Delta T_{\mathrm{C}}$ 表示靠近 冷浴的单元的温度升高量. 如图 6 所示, 实心圆形标 记代表模型 $\mathrm{A}$ 的模拟数据, 空心圆形标记代表模型 $\mathrm{B}$ 的模拟数据. 对于 2 种模型, $\Delta T_{\mathrm{C}}$ 均随 $\Delta T_{\mathrm{H}}$ 表现线性增加. 在整个 $\Delta T_{\mathrm{H}}$ 变化区间内, 模型 $\mathrm{A}$ 的 $\Delta T_{\mathrm{C}}$ 增加速率大于模 型 $\mathrm{B}$ ，表明模型 $\mathrm{A}$ 的传热性能要高于模型 $\mathrm{B}$.

图 6 中方形标记表示热整流效率 $\eta$, 右边为其纵 坐标 $\Delta T_{\mathrm{C}}$, 可以通过下式计算得到 ${ }^{[3]}$

$$
\lambda=\frac{P}{\Delta T_{\mathrm{H}}-\Delta T_{\mathrm{C}}}\left(\frac{\Delta T_{\mathrm{C}}}{\Delta T_{\mathrm{H}}+\Delta T_{\mathrm{C}}}\right),
$$

$\lambda$ 表示热导, $P$ 表示热浴加热器加热功率, 等式右边 第 2 项为功率 $P$ 利用率. 由于 $\lambda \propto \Delta T_{\mathrm{C}}$, 故在相同 $\Delta T_{\mathrm{H}}$ 下，可以通过 $\Delta T_{\mathrm{C}}$ 比较热导.

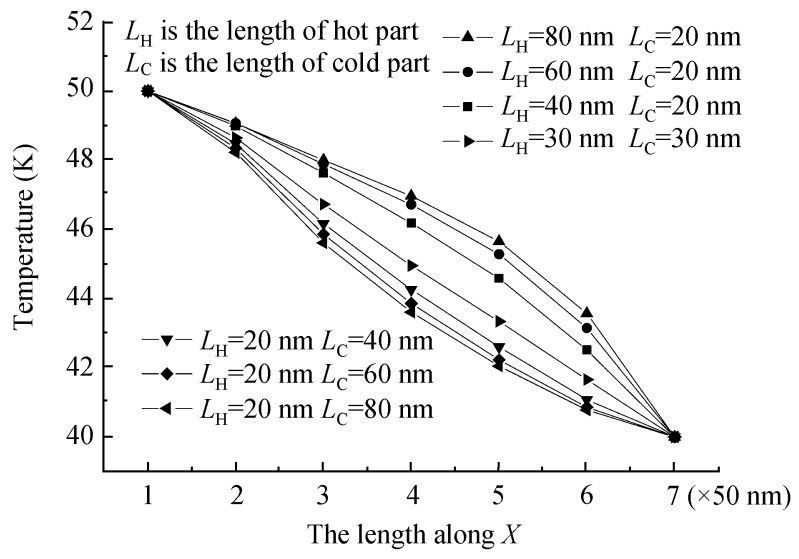

图 $5 X$ 轴方向温度分布(热端温度 $50 \mathrm{~K}$, 冷端温度 $40 \mathrm{~K}$ ) 


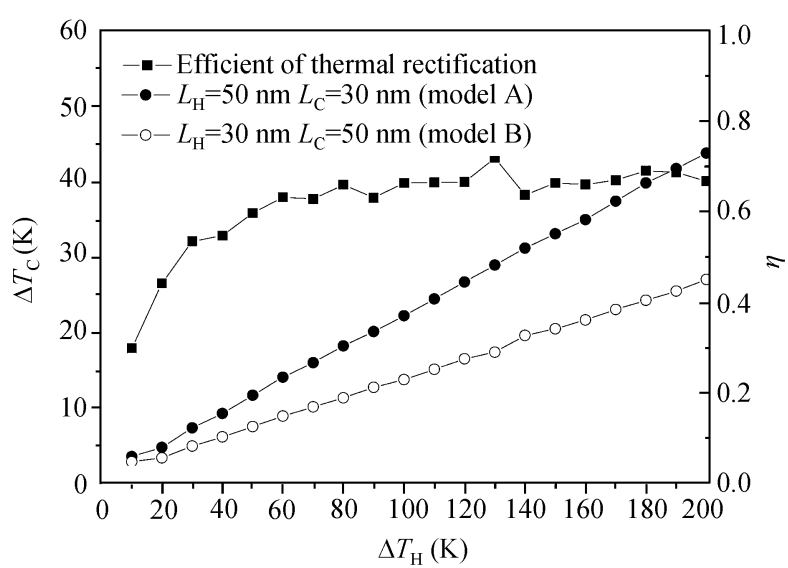

图 6 非等热量输入模型热整流效应

$$
\eta=\frac{\lambda_{\mathrm{bs}}-\lambda_{\mathrm{sb}}}{\lambda_{\mathrm{sb}}},
$$

$\lambda_{\mathrm{bs}}$ 表示模型 $\mathrm{A}$ 的热导, $\lambda_{\mathrm{sb}}$ 表示模型 $\mathrm{B}$ 的热导. $\Delta T_{\mathrm{H}}<40 \mathrm{~K}$ 时, $\eta$ 从 0.35 迅速增长至 $0.65, \Delta T_{\mathrm{H}} \geqslant 40 \mathrm{~K}$ 时, $\eta$ 在 0.65 附近震荡趋于稳定.

本文第 2 部分比较了模型 $\mathrm{A}$ 与模型 $\mathrm{B}$ 的边界散 射, 分析结果表明模型 $\mathrm{B}$ 对声子起加速作用. 然而在 图 6 中却得到模型 $\mathrm{A}$ 热导性能优于模型 $\mathrm{B}$ 的结果, 可 能是由 2 个因素造成: 1) 模型 $\mathrm{A}$ 热浴的体积大于模型 $\mathrm{B}$ 热浴的体积, 造成前者每个步长时间内输入的热流 量大于后者, 因而有必要观察等热量输入情况下 2 种 模型的热阻变化;2) 模型 $\mathrm{A}$ 中, 靠近冷浴单元的体积 小于模型 $\mathrm{B}$ 末端单元体积, 因而对于相同的热量, 模 型 $\mathrm{A}$ 末端单元温度大于模型 $\mathrm{B}$ 末端单元温度. 为了 进一步判断以上分析, 建立如图 7 所示 2 个模型, 2 个模型中热浴和冷浴的宽度相等即 $L_{\mathrm{s}}=L_{\mathrm{b}}=30 \mathrm{~nm}$, 长 度均为 $50 \mathrm{~nm}$, 厚度为 $50 \mathrm{~nm}$, 从而可以保证在相同 热浴温度下每个步长时间进入 2 个模型的热量相等.

图 8 显示了等热量输入模型的整流效应，在模型

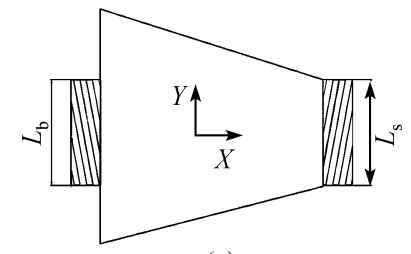

(a)

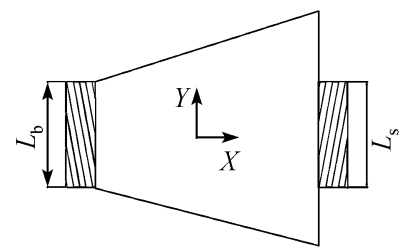

(b)
图 7 变截面纳米线示意图(等能量输入)

(a) 热浴设置在大端, 冷浴设置在小端( 记为模型 $\mathrm{A}^{\prime}$ );

(b) 热浴设置在小端, 冷浴设置在大端(记为模型 $\mathrm{B}^{\prime}$ )

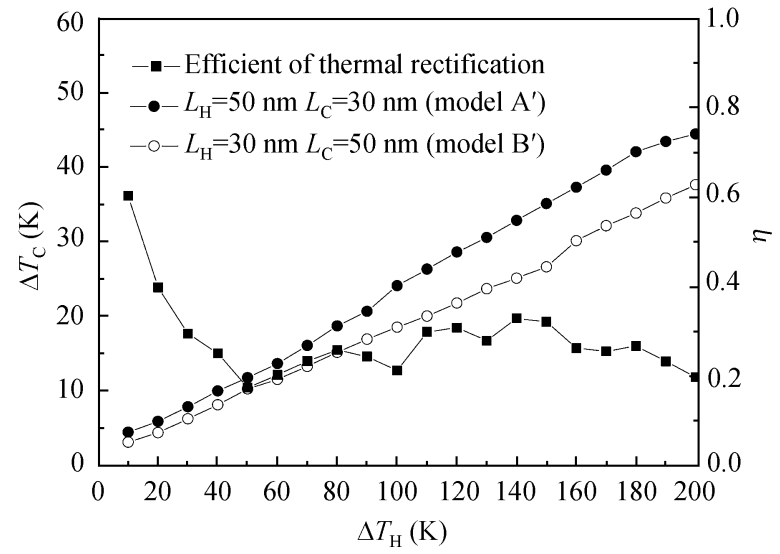

图 8 等热量输入模型的热整流效应

$\mathrm{A}^{\prime}$ 和 $\mathrm{B}^{\prime}$ 中, $\Delta T_{\mathrm{C}}$ 均随 $\Delta T_{\mathrm{H}}$ 线性增加. 与图 6 相比, 对于模 型 $\mathrm{A}^{\prime}, \Delta T_{\mathrm{C}}$ 增长率并未发生显著变化; 对于模型 $\mathrm{B}^{\prime}, \Delta T_{\mathrm{C}}$ 的增长率明显加大, 原因在于模型冷浴的体积减小, 声子由末端单元进入冷浴的通道变窄, 使一部分声子 被壁面反射回去造成的. 需要注意的是, 虽然在模拟 过程中每个步长时间内, 由 2 个模型的热浴流入的声 子相等并且模型 $\mathrm{B}^{\prime}$ 的边界散射对热流起积极作用, 但 是模型 $\mathrm{A}^{\prime}$ 末端温度在整个 $\Delta T_{\mathrm{H}}$ 变化范围内始终高于模 型 $\mathrm{B}^{\prime}$. 这表明对于相同体积的模型，几何形状不同引 起的质量分布差异对于热传导起到了重要的作用. 热 整流效率 $\eta$ 的变化曲线, 先经历了一个迅速下降的过 程, 然后在 0.2 0.35 附近震荡趋于平衡. 图 8 中, $\Delta T_{\mathrm{H}}<$ $40 \mathrm{~K}$ 范围内, $\Delta T_{\mathrm{C}}$ 数值变化的较大, 分析认为可能是由 于模型 $\mathrm{A}^{\prime}, \mathrm{B}^{\prime}$ 在此温度段内的 $\Delta T_{\mathrm{C}}$ 数值比较接近, 求解 (9)式过程中引入了计算误差造成了较大的统计涨落. 非等热量模型稳定后的热整流效率约为 $0.6 \sim 0.7$, 等热 量模型稳定后的热整流效率约为 0.2 0.3.

图 9 所示为等热量输入条件下, 2 种模型的热阻 变化曲线, 纵坐标为热阻. 由下式计算得到热阻值 ${ }^{[13]}$, 横坐标为热浴温度变化量

$$
R=\frac{\Delta T}{Q},
$$

式中, $R$ 表示热阻, $\Delta T$ 表示靠近热浴单元与靠近冷浴 单元的温度差, $Q$ 表示稳态时热流. 空心圆形标记表 示模型 $\mathrm{B}^{\prime}$ 的热阻, 实心圆形标记表示模型 $\mathrm{A}^{\prime}$ 的热阻. 在模拟温度区间内, 模型 $\mathrm{B}^{\prime}$ 的热阻始终大于模型 $\mathrm{A}^{\prime}$. 模拟结果验证了图 8 中在等热量输入条件下, 模型 $\mathrm{A}^{\prime}$ 的热导性能高于模型 $\mathrm{B}^{\prime}$ 的结论. 当 $\Delta T_{\mathrm{H}}=50 \mathrm{~K}$ 时, 模 型 $\mathrm{B}^{\prime}$ 的热阻约为模型 $\mathrm{A}^{\prime}$ 的 1.78 倍. 当 $\Delta T_{\mathrm{H}}=200 \mathrm{~K}$ 时, 


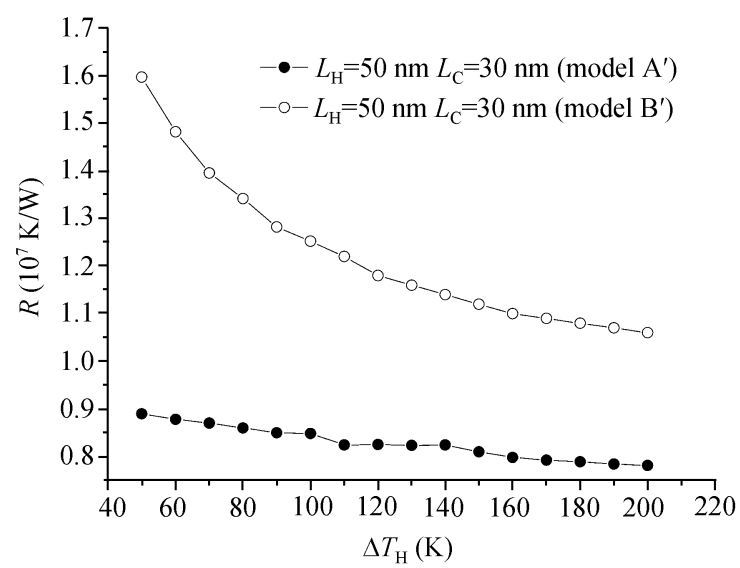

图 9 热阻随温度变化关系图

模型 $\mathrm{B}^{\prime}$ 的热阻约为模型 $\mathrm{A}^{\prime}$ 的 1.36 倍. 随着温度的增 加, 高频低速声子占总声子比例的增多导致 U 散射 发生的几率增加, 边界散射几率逐渐减小. 由于边界 效应对模型影响的权重逐渐降低, 从而引起 2 个模型 的热阻差随 $\Delta T_{\mathrm{H}}$ 增大而减小.

\section{4 结论}

采用 $\mathrm{MC}$ 方法模拟了声子在变截面纳米线结构 中的输运, 模拟比较了渐扩模型与渐缩模型在 $45 \mathrm{~K}$ 时的温度分布, 渐扩模型温度分布曲线呈下凹变化, 渐缩模型温度分布曲线呈上凸变化. 调整模型大端 与小端的尺寸比, 尺寸比越大温度分布的非线性愈 明显. 分析了边界散射对 2 种模型的影响, 认为在渐 扩模型中边界散射对于声子传输起加速作用, 对渐 缩模型的作用与之相反. 分别研究了等热量与非等 热量 2 种输入条件下, 变截面纳米线的热整流效应. 结果表明质量分布引起的体积分布不同是造成热传 导性能差异的主要因素, 同时表明边界散射对声子 输运的作用相对较弱. 在非等热量输入与等热量输 入条件下, 对于变截面纳米线前者的热整流效率大 于后者. 由此可见，纳米结构的形状对热传导的影响 具有显著作用, 利用此特性可以通过调节边界几何 形态达到控制热阻与热流的目的.

\section{参考文献}

1 Li D Y, Wu Y Y, Kin P, et al. Thermal conductivity of individual silicon nanowires. Appl Phys Lett, 2003, 83(14): 2934-2396

2 Hochbaum A I, Chen R K, Delgado R D, et al. Enhanced thermoelectric performance of rough silicon nanowires. Nature, 2008, 451(7175): $163-167$

3 Chang C W, Okawa D, Majumdar A, et al. Solid-state thermal rectifier. Science, 2006, 314(5802): 1121-1123

4 Wu G, Li B W. Thermal rectification in carbon nanotube intramolecular junctions: Molecular dynamics calculations. Phys Rev B, 2007, 76(8): $085424-085431$

5 Yang N, Zhang G, Li B W. Carbon nanocone: A promising thermal rectifier. Appl Phys Lett, 2008, 93(24): 243111

6 Lü X, Chu J H, Shen W Z, et al. Modification of the lattice thermal conductivity in semiconductor rectangular nanowire. J Appl Phys, 2003, 93(2): $1219-1229$

7 Allen M P, Tildesley D J. Computer Simulation of Liquids. Oxford: Clarendon Press, 1987

8 Chen Y F, Li D Y, Lukes J R, et al. Monte Carlo simulation of silicon nanowire thermal conductivity. J Heat Transfer, 2005, 127(10): 11291137

9 Lacroix D, Joulain K, Lemonnier D, et al. Monte Carlo transient phonon transport in silicon and germanium at nanoscales. Phys Rev B, 2005, 72(6): 064305-064315

10 Lacroix D, Joulain K, Terris D, et al. Monte Carlo simulation of phonon confinement in silicon nanostructures: Application to the determination of the thermal conductivity of silicon nanowires. Appl Phys Lett, 2006, 89(10): 103104-103106

11 Mazumder S, Majumdar A. Monte Carlo study of phonon transport in solid thin films including dispersion and polarization. J Heat Transfer, 2001, 123(5): 749-759

12 Kittel C. Introduction to Solid State Physics. New York: John Wiley \& Sons Inc, 1986

13 Cao B Y, Guo Z Y. Heat conduction in vanostructures with variable cross-section. J Eng Thermophys, 2007, 28(6): 983-985 